\title{
Simulation of Spacecraft Fuel Tank Self-pressurization Using Eulerian-Lagrangian Method
}

\author{
Jaeheon Sim $^{*}$ and Chih-Kuang Kuan ${ }^{\dagger}$ \\ University of Michigan, Ann Arbor, MI, 48109 \\ and \\ Wei Shyy" \\ University of Michigan, Ann Arbor, MI, 48109 \\ Hong Kong University of Science and Technology, Kowloon, Hong Kong
}

\begin{abstract}
A 3-D adaptive Eulerian-Lagrangian method is further implemented with a phase change model to study the thermal effect in a spacecraft fuel tank, especially the self-pressurization of a cryogenic propellant tank. The stationary (Eulerian) frame is used to resolve the flow field, and the marker-based triangulated moving (Lagrangian) surface meshes are utilized to treat fluid interfaces and solid boundaries. The main focus of the present study is to improve the accuracy of the sharp solid boundary treatment and to implement a phase change model. The energy and mass transfer across the interface due to phase change is computed from Stefan condition using probe-based temperature gradient computations. Uniform flow past a cylinder, 1-D Stefan problems, and 2-D melting cases by natural convection flow are presented for validation purpose of the present approaches. The self-pressurization in a liquid hydrogen fuel tank is simulated and it is shown that the conduction-only solution underestimates the pressure rise, and the full Navier-Stokes and energy equation solution is required to study liquid fuel tank pressurization due to the influence of transport phenomena.
\end{abstract}

\section{Introduction}

SPACE missions - including rocket launchers, the space stations, and interplanetary space flights - require an understanding and managing of the cryogenic liquid propellants under varying acceleration conditions. This understanding is crucial because cryogenic liquid dynamics have a significant impact on the engine operation, vehicle dynamics, spacecraft design, and even the overall mission. Moreover, the thermal effect in a cryogenic propellant has huge influence on the spacecraft safety as well as fuel dynamics. Even small heat leak from incident solar radiation, aerodynamics heating, or spacecraft structure causes thermal stratification and fuel vaporization since the boiling temperature of cryogenic propellant is extremely low, and usual space missions require a long operational time. The fuel vaporization results in cryogenic propellant loss and self-pressurization in a fuel tank, and this determines the design safety of a fuel tank as well as the operational time and the required fuel amount.

It is known that the cryogenic vaporization due to filling process and heat leak causes mass loss and selfpressurization in the storage tank, and improving the fuel tank storage and lower heat transfer results in significant cost savings through reduction of launch mass. ${ }^{1}$ Many researchers have conducted studies on the cryogenic storage tank under normal and micro-gravity conditions. Aydelott showed the rate of pressure rise is lower in reducedgravity than normal gravity because of the increased liquid-wetted wall area. ${ }^{2}$ Poth and Van Hook showed that a mixing jet could be used to minimize thermal stratification and reduce pressure rise in a tank. ${ }^{3}$ Application of subcooled jet mixing is further studied experimentally, and both passive insulation and active cryocooler technology is now implemented to reduce self-pressurization. ${ }^{4-6}$ The numerical studies followed for deeper understanding and

\footnotetext{
* Post-doctoral Research Fellow, Department of Aerospace Engineering, University of Michigan; AIAA member.

${ }^{\dagger}$ Graduate Student, Department of Aerospace Engineering, University of Michigan; AIAA Student member.

"Clarence L. "Kelly" Johnson Collegiate Professor, Department of Aerospace Engineering, University of Michigan; currently, Provost \& Chair Professor, Department of Mechanical Engineering, Hong Kong University of Science and Technology; AIAA Fellow.
} 
overcome the experimental limitation. However, due to its complexity including flow motions, heat transfer, and phase change, the numerical and theoretical studies are conducted in a limited condition. Many researchers just focus on the thermal effect in cryogenic liquid region by assuming homogenous condition in vapor phases using a lumped thermodynamic model of vapor region. ${ }^{7,8}$ Another popular topic is the study on the mixing jet and thermal distributions in a liquid region by ignoring phase change and self-pressurization. ${ }^{9-11}$

Considering microgravity conditions in a typical spacecraft environment, the capillary effect becomes very important due to small Bond number, the ratio of body (or gravitational) forces to surface tension forces. Thus, a study on the multiphase flow including interfacial dynamics is required in order to understand the liquid fuel dynamics in a spacecraft. However, experimental studies are limited because the microgravity conditions are hard to realize on the ground. Drop tower tests and in-flight tests have been conducted, but their short operational times prohibited simulating practical engineering problems. Thus, a high fidelity numerical simulation of such a multiphase flow in a space environment is crucial to compensate the limitation of experiment. However, the numerical simulations of such problems are also challenging due to multiple time/length scales, large variations in fluid properties, moving boundaries, and phase changes.

The multiphase flow includes an interface between different phases having distinct physical properties, and consequently involves identifying interface location and modeling interfacial dynamics, including a steep jump in fluid properties in response to surface tension effects. Various methods have been proposed and improved and each method has its own relative strengths and weaknesses. ${ }^{12,13}$

In order to represent interface in moving boundary computations, three main categories exist as reviewed by Shyy et al.; ${ }^{12}$ Lagrangian (moving grid), Eulerian (stationary grid), and Eulerian-Lagrangian methods. The Lagrangian method uses body-fitted grid for tracking interfaces, and is accurate since interfacial conditions can be applied at the exact interface location. However, maintaining grid quality is difficult, and computationally expensive to use when large deformation or movements occur. The Eulerian (stationary grid) method extracts the interface location via post processing with a help of a scalar function on a stationary computational grid, and its simplicity and natural handling of topological change makes these methods very popular. However, implicit interface extraction causes errors in interface location or surface tension computation. Eulerian-Lagrangian method utilizes a separate set of moving (Lagrangian) mesh and associated marker/tag system representing interface on a stationary (Eulerian) grid used to compute the flow fields. Thus, the interface can be tracked explicitly, and these methods possess several desirable features when compared with Lagrangian or Eulerian method.

Another challenging issue is handling of the interfacial dynamics even if we know the location and shape of the interface at a given time. Across an interface, in general, the pressure and viscous stresses show discontinuities and fluid properties suddenly jump due to surface tension. Moreover, phase change includes mass and heat transfer across an interface. How to treat such a phase discontinuity and associated capillary effects can be classified into two categories: the sharp interface method and the continuous interface method. The sharp interface method is a class of techniques which satisfy the jump condition across phase boundary exactly on the interface of zero thickness. Compared to the sharp interface method, the continuous interface method smoothes out the fluid properties and interfacial forces around phase boundary instead of a zero thickness interface. A popular and highly successful continuous interface technique is the immersed boundary method proposed by Peskin. ${ }^{14}$ It utilizes a single fluid formulation for the entire domain by smoothing out the fluid property and modeling the surface tension forces as a momentum source term within finite range. The continuous interface method is implemented successfully by many researchers to simulate large deformable or merger/breakup fluid flows. ${ }^{15,16}$ Ye et al. presented a sharp interface approach based on the Eulerian-Lagrangian approach, which treats the interfacial physics without smearing. ${ }^{17,18}$ Mittal et al. further implemented a sharp interface method with the help of ghost cells by reconstructing the solution on those to satisfy the jump condition across interface. ${ }^{19}$ The cut-cell and ghost fluid methods belong to this category. Usually, a fluid/solid interface is modeled by a sharp interface method due to small deformation and there is no need to solve solid phase while fluid/fluid interface is modeled by a continuous method because it is easier to implement and has better capability in handling various multiphase problems even with large deformation and moving interfaces.

The phase change and following mass transfer across phase boundary has not been well understood due to its physical and numerical complexity. Son et al. solved the boiling bubbles problem using the level set method. They assumed the temperature inside the bubble to be constant and computed mass transfer with temperature gradient only at the liquid side. ${ }^{20}$ This assumption is applied with the VOF method by Welch and Wilson. ${ }^{21}$ Juric and Tryggvason extended an immersed boundary method into a boiling problem by adding a smoothed latent heat source term into the energy equation. They iterated the whole solution procedure estimating the amount of mass transfer ${ }^{22}$ Shin and Juric simplify the method of computing the mass transfer from the known temperature gradient, but at both the liquid and the vapor sides. ${ }^{23}$ However, continuous treatment with diffused material property of thermal 
conductivity limited the accuracy of heat transfer across the interface. Luo et al. implemented a hybrid method using sharp treatment for temperature computation with a standard continuous level-set method. ${ }^{24}$ Morgan improved accuracy in a phase change computation by correcting the temperature around the interface and directly using linear interpolation based on the interface temperature. This hybrid approach is very popular due to its simplicity and accuracy. However, the material properties are computed differently for momentum equations and the energy equation, and the sharp treatment is not appropriate for large deformable interfacial flows. Ferziger conducted an interesting study to demonstrate that the errors in continuous temperature treatment may be from the incorrect smoothed average of thermal conductivity around the interface in steady pure heat conduction problem with no phase change. ${ }^{25}$

In the present study, we have further developed the previously reported 3-D adaptive Eulerian-Lagrangian method $^{16,26,27}$ by implementing both continuous and sharp interface methods for energy equation with phase change effect. This numerical method utilizes the stationary (Eulerian) frame to resolve the flow field, and the marker-based triangulated moving (Lagrangian) surface meshes to treat the phase boundary interfaces. The large-deformable fluid boundaries are modeled using a continuous interface method, and the surface tension between fluid interfaces is smeared within finite distance. The solid boundaries are treated by a sharp interface method along with the ghost cell method by reconstructing the solution on the ghost cell based on the known solid boundary condition. The contact line where the fluid interface meets the solid boundary is modeled using a contact line force model, which enforces the given contact angle dynamically. For moving contact line treatment, local slip condition is applied around the contact line. The adaptive Cartesian grid method is implemented to resolve the sufficient computation resolution, especially around interface with effective computation. The energy equation solver is added into the previous study, and Boussinesq approximation is used for Buoyancy force computation in an incompressible flow solver. The phase change model using continuous treatment for temperature is developed with modified smoothing of material properties. 1-D Stefan problems and 2-D melting case by convection/diffusion flow are presented for validation purpose of the present approaches. The self-pressurization in a liquid hydrogen fuel tank under normal gravity is simulated with various heat distributions and liquid fill-levels.

\section{Numerical Methods}

In the present study, the marker-based 3-D adaptive Eulerian-Lagrangian method is further implemented to perform interfacial flow computations including phase change. The bulk flow variables are solved on the stationary (Eulerian) background grid, whereas interface variables are handled by moving (Lagrangian) surface meshes. Figure 1 shows brief illustration of the present Eulerian-Lagrangian method, and detailed numerical method can be found in the previous works. ${ }^{16,26,27}$

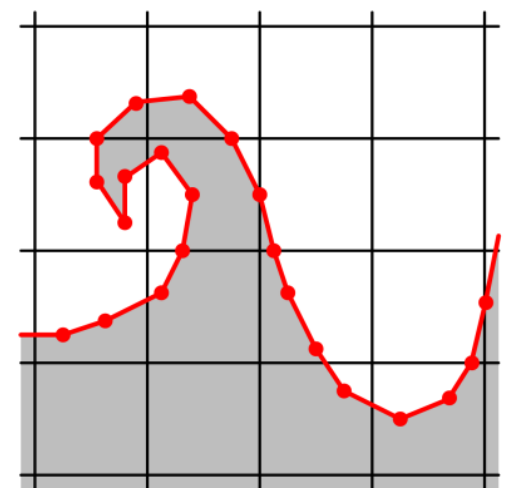

(a)

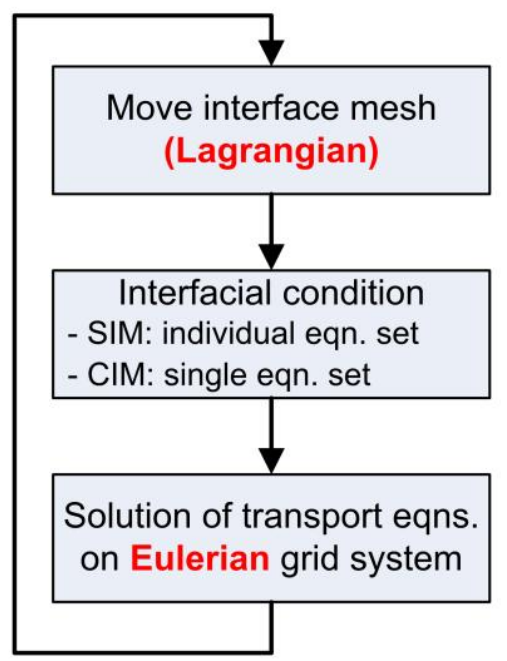

(b)

Figure 1. Eulerian-Lagrangian methods. (a) Interface representation and tracking using moving meshes on the stationary Cartesian grid. (b) Solution procedures. 


\section{A. Governing equations}

A single fluid formulation for all fluid phases is made possible by smoothing out the properties across the interface. Non-dimensionalized incompressible Navier-Stokes equations for mass and momentum conservation are given in Eqs. (1) and (2) respectively, which accounts for the interfacial dynamics as source terms in the governing equations; surface tension effects of fluid interfaces as a momentum forcing term $\left(\boldsymbol{F}_{f}\right)$, and the latent heat effects across fluid interfaces as a energy source term $\left(Q_{f}\right)$. In Eqs. (1) and (2), $\boldsymbol{u}$ is the velocity vector, and $\rho, \mu$, and $p$ is the density, viscosity, and pressure, respectively.

$$
\begin{gathered}
\frac{\partial \rho}{\partial t}+\boldsymbol{\nabla} \cdot(\rho \boldsymbol{u})=0 \\
\frac{\partial \rho \boldsymbol{u}}{\partial t}+\boldsymbol{\nabla} \cdot(\rho \boldsymbol{u} \boldsymbol{u})=-\boldsymbol{\nabla} p+\frac{1}{R e} \boldsymbol{\nabla} \cdot\left(\mu \boldsymbol{\nabla} \boldsymbol{u}+\mu \boldsymbol{\nabla}^{T} \boldsymbol{u}\right)+\frac{1}{F r} \rho \boldsymbol{g}+\frac{1}{W e} \boldsymbol{F}_{f} \\
\frac{\partial \rho C T}{\partial t}+\boldsymbol{\nabla} \cdot(\rho C T \boldsymbol{u})=\boldsymbol{\nabla} \cdot(K \boldsymbol{\nabla} T)+Q_{f}
\end{gathered}
$$

Here, all variables are non-dimensionalized by a characteristic velocity (U) and length scale (L), standard gravity $\left(\mathrm{g}_{0}\right)$, and liquid material properties (density $\rho_{l}$, viscosity $\mu_{l}$, and surface tension $\sigma$ ). The non-dimensional parameters of Reynolds, Froude, and Weber numbers in Eq. (2) are defined as, $\operatorname{Re}=\rho_{l} \mathrm{UL} / \mu_{l}, \mathrm{Fr}=\mathrm{U}^{2} /\left(\mathrm{g}_{0} \mathrm{~L}\right)$, and We $=$ $\rho_{l} \mathrm{U}^{2} \mathrm{~L} / \sigma$. In addition, the capillary number and bond number can be defined as $\mathrm{Ca}=\mathrm{We} / \mathrm{Re}=\mu_{l} \mathrm{U} / \sigma$ and $\mathrm{Bo}=$ $\mathrm{We} / \mathrm{Fr}=\rho_{l} \mathrm{~g}_{0} \mathrm{~L}^{2} / \sigma$, respectively.

The single set of equations formulation for all fluid phases in the whole domain is achieved with the help of an approximate Dirac delta function $\left(\delta_{h}\right)$ and an indicator function $(I)$. The approximate Dirac delta function, originally proposed by Peskin ${ }^{14}$ is implemented over finite thickness of 4 cell width instead of the analytical form of Dirac delta function, which has a non-zero value only at the zero-thickness interface. The Indicator function is a scalar function varying from zero to one smoothly across the interface and has a value of 0.5 at the interface location. The smoothed fluid properties such as density and viscosity are computed using Eqs. (4)-(7).

$$
\begin{gathered}
\rho=\rho_{2}+\left(\rho_{1}-\rho_{2}\right) I \\
\frac{\rho}{\mu}=\left(\frac{\rho}{\mu}\right)_{2}+\left[\left(\frac{\rho}{\mu}\right)_{1}-\left(\frac{\rho}{\mu}\right)_{2}\right] I \\
\rho C=(\rho C)_{2}+\left[(\rho C)_{1}-(\rho C)_{2}\right] I \\
\frac{\rho C}{k}=\left(\frac{\rho C}{k}\right)_{2}+\left[\left(\frac{\rho C}{k}\right)_{1}-\left(\frac{\rho C}{k}\right)_{2}\right] I
\end{gathered}
$$

Separate indicator functions for fluid and solid interface are used to separate the designation of solid interface, modeled by a sharp interface method, from fluid interface modeled by a continuous interface method. The indicator function is computed using a discrete form of the Heaviside step function in Eq. (8) by integrating 1-D form of discrete Dirac delta function. This approach is known being applicable more generally than Poisson equation solver method since it requires only distance information from interface, and thus gives accurate values even at the boundaries. ${ }^{26}$

$$
I(\mathbf{x})=\mathrm{H}(\mathrm{r}=\mathbf{n} \cdot(\mathbf{x}-\mathbf{X}))=\int_{-\infty}^{\mathrm{r}} \delta(\mathrm{h}) \mathrm{dh}
$$




\section{B. Solution procedure: Projection Method}

The governing equations (1)-(3) are solved using a projection method with a staggered grid finite volume formulation. The pressure and fluid properties are stored at the cell center and face-normal velocities are stored on the Cartesian cell faces. The convection term $(\boldsymbol{N})$ is discretized using a 3rd order ENO scheme in space and a 2nd order Runge-Kutta integration in time. The central difference scheme and Crank-Nicholson method is implemented for the viscous term $(\boldsymbol{L})$. The discretized solution procedures are summarized in Eqs. (9)-(12). In Eq. (13), $\nabla \cdot \boldsymbol{u}^{n+1}$ term is zero if the phase change doesn't occur and mass is not transferred across the interface.

$$
\begin{gathered}
\frac{T^{n+1}-T^{n}}{\Delta t}=-\nabla \cdot(\boldsymbol{u} T)^{n+1 / 2}+\frac{1}{(\rho C)^{n+1}}\left[\nabla \cdot(K \nabla T)^{n+1}+Q_{f}^{n+1}\right] \\
\frac{\boldsymbol{u}^{*}-\boldsymbol{u}^{n}}{\Delta t}=-\boldsymbol{N}\left(\boldsymbol{u}^{n+1 / 2}\right)+\frac{1}{\rho^{n+1}}\left[-\nabla p^{n}+\boldsymbol{L}\left(\boldsymbol{u}^{n+1 / 2}\right)+\boldsymbol{F}^{n+1}\right] \\
\boldsymbol{u}^{* *}=\boldsymbol{u}^{*}+\frac{\Delta t}{\rho^{n+1}} \nabla p^{n} \\
\boldsymbol{u}^{n+1}=\boldsymbol{u}^{* *}-\frac{\Delta t}{\rho^{n+1}} \nabla p^{n+1}
\end{gathered}
$$

with Pressure Poisson equation of Eq. (13) by taking divergence of Eq. (12).

$$
\nabla^{2}\left(p^{n+1}\right)=-\frac{\Delta t}{\rho^{n+1}}\left(\nabla \cdot \boldsymbol{u}^{* *}-\nabla \cdot \boldsymbol{u}^{n+1}\right)
$$

\section{Interface representation and tracking}

In this marker-based Eulerian-Lagrangian method, the interface is represented by massless markers in coordination with each other for maintaining the interface connectivity information. The corresponding data structure is established via line-segments in two-dimensional and triangles in three-dimensional domains, as represented in Figure 1(a). The marker locations, denoted by $\mathbf{X}$ in Lagrangian frame, are updated from the velocities at its location, $\mathbf{V}(\mathbf{X})$, in Eq. (14).

$$
\frac{\partial \mathbf{X}}{\partial \mathrm{t}}=\mathbf{V}(\mathbf{X})
$$

Fluid interfaces use the computed flow solution field to obtain the marker velocities as shown in Eq. (15). In this equation, the discrete Dirac delta function, $\delta(\mathbf{x}-\mathbf{X})$, is employed for converting the Eulerian velocity field, $\mathbf{u}(\mathbf{x})$, to Lagrangian form, $\mathbf{U}(\mathbf{X})$. The interface velocity is exactly same to fluid velocity if there is no mass transfer in Eq. (15). However, with phase change, the velocity component from mass transfer should be considered. On the other hand, solid interfaces use the prescribed velocity field to advance the marker points using Eq. (14).

$$
\boldsymbol{U}(\boldsymbol{X})=\int_{V} \boldsymbol{u}(\boldsymbol{x}) \delta(\boldsymbol{x}-\boldsymbol{X}) d V-\frac{\dot{m}}{\rho_{f}} \cdot \boldsymbol{n}
$$

In order to maintain consistent computational accuracy, the spacing between marker points is rearranged by adding or deleting markers whenever two markers come too close or too distant from each other. The level-contourbased interface reconstruction technique with connectivity information is also implemented to handle topological changes such as merger or break-up. Detailed numerical method can be found in the previous works. ${ }^{16,26,27}$ 


\section{Interfacial dynamics modeling}

The continuous interface method is implemented for all fluid phases by a single fluid formulation due to its effectiveness in modeling highly deformable fluid interfaces. The surface force computation of Eq. (16), where $\sigma$ is the surface tension and $\kappa$ is the curvature of interface, is applied as source term in the momentum equation, Eq. (2).

$$
\boldsymbol{F}_{f}=\int_{\Gamma(t)} \sigma \kappa \delta(\boldsymbol{x}-\boldsymbol{X}) d \Gamma
$$

Solid interfaces is modeled using the sharp interface method by reconstructing solution fields around an interface to incorporate the given boundary condition on the solid interface in an Eulerian Cartesian grid. The velocity reconstruction on the solid side is implemented via ghost cells, which are defined as solid cells having at least one neighboring fluid cell. This approach works well even with contact line where fluid interface meets solid interface.

When a fluid-fluid interface intersecting a solid surface, the treatment of the tri-junction locations, called contact line, is required to account for the presence and interactions of all three phases, fluid-fluid-solid. One of the most discussed issues for modeling these contact lines with Navier-Stokes equations is that the imposed no-slip condition for velocity leads to a non-integrable singularity in stress. In the present research, the contact line force is imposed with local slip condition to overcome this singularity issue.

The basic idea of these two different approaches is shown in Figure 2, and the details can be found in our previous works. ${ }^{16,26,27}$

\section{E. Improvement of solid boundary treatment}

In the present sharp interface method for solid boundary treatment, the boundary condition of solid surface is enforced through the usage of ghost cells which are defined as solid cells having at least one neighboring fluid cells. Thus, the accuracy is directly related to the accuracy of interpolations at ghost cells. In Figure 3, the quadratic interpolation is implemented with two additional imaginary points (IP) in fluid phase instead of the previous linear interpolation with just one imaginary point.

Two imaginary points, IP1 and IP2 are built by extrapolating the normal of the interface originated from a ghost cell toward the fluid phase with a constant interval while the solid point (SP) is the intersection point of the normal and interface. The minimum cell width is used as the interval for SP to IP1 and IP1 to IP2. Bi/tri-linear interpolation is applied to determine the values at the imaginary points. Four cells with square stencil are implemented for bilinear reconstruction in two-dimensional domain and eight cells with cubic stencil for tri-linear interpolation in threedimensional domain. As shown in Figure 3, velocity of IP1 is reconstructed based on chosen cells marked by red square dash line and so as the green square for IP2. An algorithm that guarantees such a geometric pattern is necessary for every image point that needs bi/tri-linear interpolation. The formulas of bi-linear and tri-linear interpolation are

$$
\begin{gathered}
U_{i}=c_{0}+c_{1} x+c_{2} y+c_{3} x y \\
U_{i}=c_{0}+c_{1} x+c_{2} y+c_{3} z+c_{4} x y+c_{5} x z+c_{6} y z+c_{7} x y z
\end{gathered}
$$

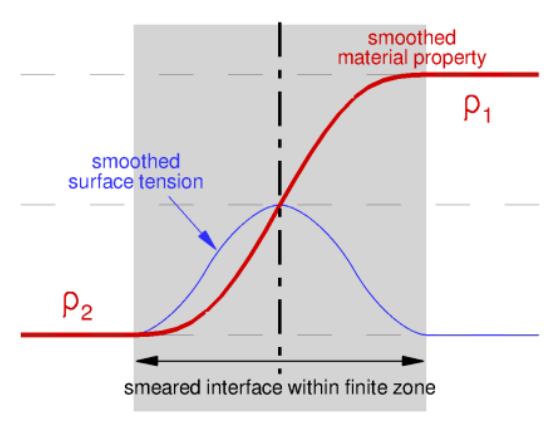

(a)

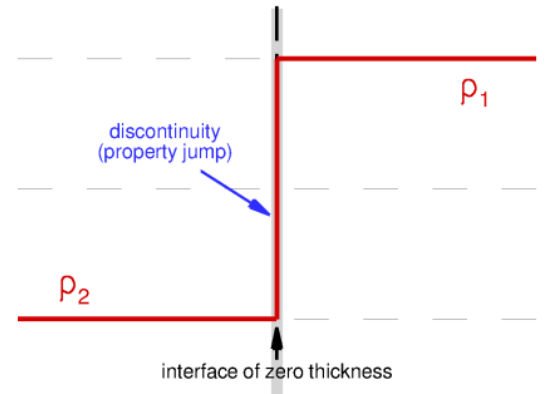

(b)

Figure 2. Interfacial dynamics modeling. (a) Fluid interface: continuous interface method with smeared interface within finite zone. (b) Solid interface: sharp interface method with the zero-thickness interface. 
Once the interpolated values at IP1 and IP2 are available, the next step is to build a quadratic representation of variables at the normal line of interface, a one-dimensional line composed by SP, IP1, and IP2. With the no-slip condition at SP and information at IP1 and IP2, quadratic formula with three coefficients is

$$
U(X)=a_{0}+a_{1} X+a_{2} X^{2}
$$

The velocity at ghost cell can be extrapolated by Eq. (19) directly with the known distance between SP and ghost cell. The conservative way is using linear extrapolation through the gradient of $\mathrm{U}(\mathrm{X})$ at $\mathrm{SP}$ as shown in Figure 3(b). Ye et al. presented quadratic reconstruction method by single image point to approximate second-order accurate boundary condition. ${ }^{17}$ It requires at least 6 cells for two-dimensional domain and 10 cells for three-dimensional domain. Sometimes it is difficult to establish appropriate multi-points stencil for interpolation especially when the image point is close to solid interface. Bi/tri-linear reconstruction is relatively easy to determine a suitable interpolation stencil in comparison to quadratic reconstruction. Moreover, utilizing two IP points whose variables are reconstructed by bi-linear interpolation to build a one-dimensional quadratic representation provide flexible boundary treatment with 2 nd order accurate approximation in at the normal of interface.

Another issue most discussed for modeling sharp interface method is mass/energy conservation on the boundary cells. Solid boundary which is not aligned with grid causes confliction between no-slip condition on the surface and mass conservation of boundary cells. Many researchers implemented finite difference method instead of finite volume method in order to detour such an issue. ${ }^{19}$ Mass/energy source/sink approach was proposed to compensate non-divergence free condition of boundary cells, but it just guarantees convergence of pressure Poisson solver, and the mass is still not conserved with computed no-slip conditions. ${ }^{28}$ Local correction method, which is our previous approach, is also a kind of mass source/sink method, and has same issues on the accuracy. ${ }^{26}$ When the Reynolds number is small and grid is fine, the velocity fields are almost steady around surface, and the error can be negligible. However, high Reynolds or Rayleigh number flow exacerbates the accuracy. In the present study, pressure boundary condition is implemented for accurate mass/energy flux computation instead of velocity boundary condition during solving pressure Poisson equation in the Projection method.

Our current approach imposes Neumann pressure boundary condition implicitly in Poisson equation. When solving the pressure Poisson equation, pressure of ghost cells is used to enforce zero normal pressure gradients at the immersed solid wall. The zero normal pressure gradients is equivalent to non-permeable wall, and thus mass conservation should be satisfied for fluid cells if ghost cell pressure is updated consistently. For each ghost cell, Neumann condition means ghost cell pressure is equal to that of the respective imaginary point. Hence, the ghost cell pressure of imaginary point could be determined by a weighting combination of multiple fluid cells adjunct to its respective image point. In a three-dimensional domain, pressure at an imaginary point is represented by a linear function of its coordinate vector $[1 \mathrm{x} \mathrm{y} \mathrm{z}]$ and so as its surrounding fluid cells.

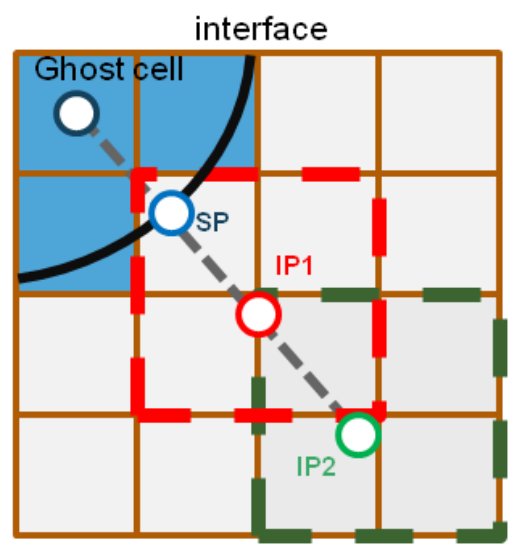

(a)

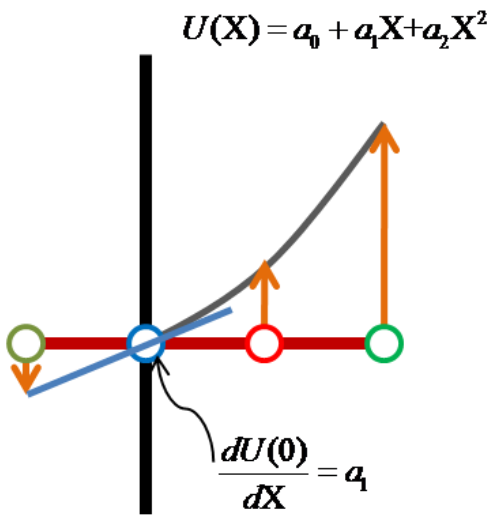

(b)

Figure 3. Quadratic interpolation scheme with two imaginary points (IP). (a) Schematics of reconstruction stencil (b) One-dimensional quadratic interpolation for a ghost cell. 


$$
P_{I P}=\left[\begin{array}{llll}
1 & X_{I} & Y_{I} & Z_{I}
\end{array}\right]\left[\begin{array}{l}
a_{1} \\
a_{2} \\
a_{3} \\
a_{4}
\end{array}\right] ; \quad\left[\begin{array}{l}
P_{1} \\
P_{2} \\
P_{3} \\
P_{4}
\end{array}\right]=\left[\begin{array}{llll}
\mathbf{1} & \boldsymbol{Y} & \mathbf{Z}
\end{array}\right]\left[\begin{array}{l}
a_{1} \\
a_{2} \\
a_{3} \\
a_{4}
\end{array}\right]
$$

In the iterative solving procedure of Poisson equation, the P1 to P4 are unknown objects. With the inverse of coordinate matrix [ $1 \mathrm{X} \mathrm{Y} \mathrm{Z}$ ], a ghost cell pressure is associated linearly to its respective fluid cells with zero wall normal derivative enforced.

$$
P_{G}=P_{I P}=\left[\begin{array}{llll}
1 & X_{I} & Y_{I} & Z_{I}
\end{array}\right]\left[\begin{array}{llll}
\mathbf{1} & \boldsymbol{X} & \boldsymbol{Z}
\end{array}\right]^{-1}\left[\begin{array}{l}
P_{1} \\
P_{2} \\
P_{3} \\
P_{4}
\end{array}\right]=\sum_{k=1}^{4} b_{k} P_{k}
$$

The discretized Poisson equation together with embedded Neumann boundary condition is solved by bistabilized conjugate gradient solver with diagonal preconditioning.

\section{F. Mass transfer due to phase change}

Mass transfer computation is one of the key issues in the phase change process since it is related to the movement of phase boundary and the amount of latent heat in energy transfer process. In the energy equation of Eq. (3), the latent heat is computed by Eq. (22), where $\dot{m}_{f}$ is mass transfer due to phase change and $L$ is the latent heat.

$$
Q_{f}=\int_{\Gamma} \dot{m}_{f} L \delta(\boldsymbol{x}-\boldsymbol{X}) d \Gamma
$$

Similar to the transformation of surface tension, the latent heat of the interface due to phase change is also transformed from a Lagrangian quantity $(\boldsymbol{X})$ to an Eulerian quantity $(\boldsymbol{x})$ via the approximate discrete Dirac delta function, $\delta_{h}(\boldsymbol{x}-\boldsymbol{X})$.

In the present study, the mass transfer is computed in Eq. (23) based on the Stefan condition using the temperature gradient with discontinuous material properties for simplicity and accuracy. Where, $\mathrm{L}$ is latent heat and $\mathrm{k}$ is thermal conductivity. The interface temperature is assumed equal to the saturation temperature since it is an adequate assumption in macroscopic problems. ${ }^{22}$

$$
\dot{m}_{f} L=\left(q_{1}-q_{2}\right) \cdot \hat{n}=-\left.k_{1} \frac{\partial T}{\partial n}\right|_{1}+\left.k_{2} \frac{\partial T}{\partial n}\right|_{2}
$$

The energy equation of Eq. (3) is solved using continuous treatment with smoothed material properties in Eqs. (4)-(7), and the projection method in Eqs. (9)-(13) is applied to solve momentum equation. The mass conservation equation in Eq. (1) is coupled by assuming $\nabla \cdot \boldsymbol{u}^{n+1}=0$ when we solve pressure Poisson equation in Eq. (13). However, the divergence of velocity is not zero around the interface if the phase change occurs and mass is transferred across the interface. Shin and Juric developed the conservation of mass in Eq. (24), ${ }^{23}$ and in the present work, the modified version of Eq. (25) is implemented for the non-conservative form of equation.

$$
\begin{gathered}
\nabla \cdot(\rho \boldsymbol{u})=\boldsymbol{u} \cdot \nabla \rho+\frac{\left(\rho_{2}-\rho_{1}\right)}{\rho_{f}} \int_{\Gamma(t)} \dot{m}_{f} \delta(\boldsymbol{x}-\boldsymbol{X}) d \Gamma \\
\nabla \cdot \boldsymbol{u}=\frac{1}{\rho}\left[\left(\rho_{2}-\rho_{1}\right) \frac{\dot{m}_{f}}{\rho_{f}} \nabla I\right]=\frac{1}{\rho}\left[\frac{\left(\rho_{2}-\rho_{1}\right)}{\rho_{f}} \int_{\Gamma(t)} \dot{m}_{f} \delta(\boldsymbol{x}-\boldsymbol{X}) d \Gamma\right]
\end{gathered}
$$




\section{G. Adaptive Grid}

Multiphase flow problems involve multiple length scales. In order to effectively resolve the flow features in such cases, adaptive grid based on isotropic refinement is implemented. The cells are split into four and eight equal sibling cells in two- and three-dimensions, respectively to better handle regions which require higher resolution. The grid is represented using unstructured data that connects cells through cell faces. The details of the algorithm can be found in Singh and Shyy. ${ }^{16}$ Adaptation is performed based on the flow solution quality for effective computation as well as the interface location.

\section{H. Overview of the present numerical method}

Figure 4 shows the schematic drawing of the present approach for multiphase flow computation.

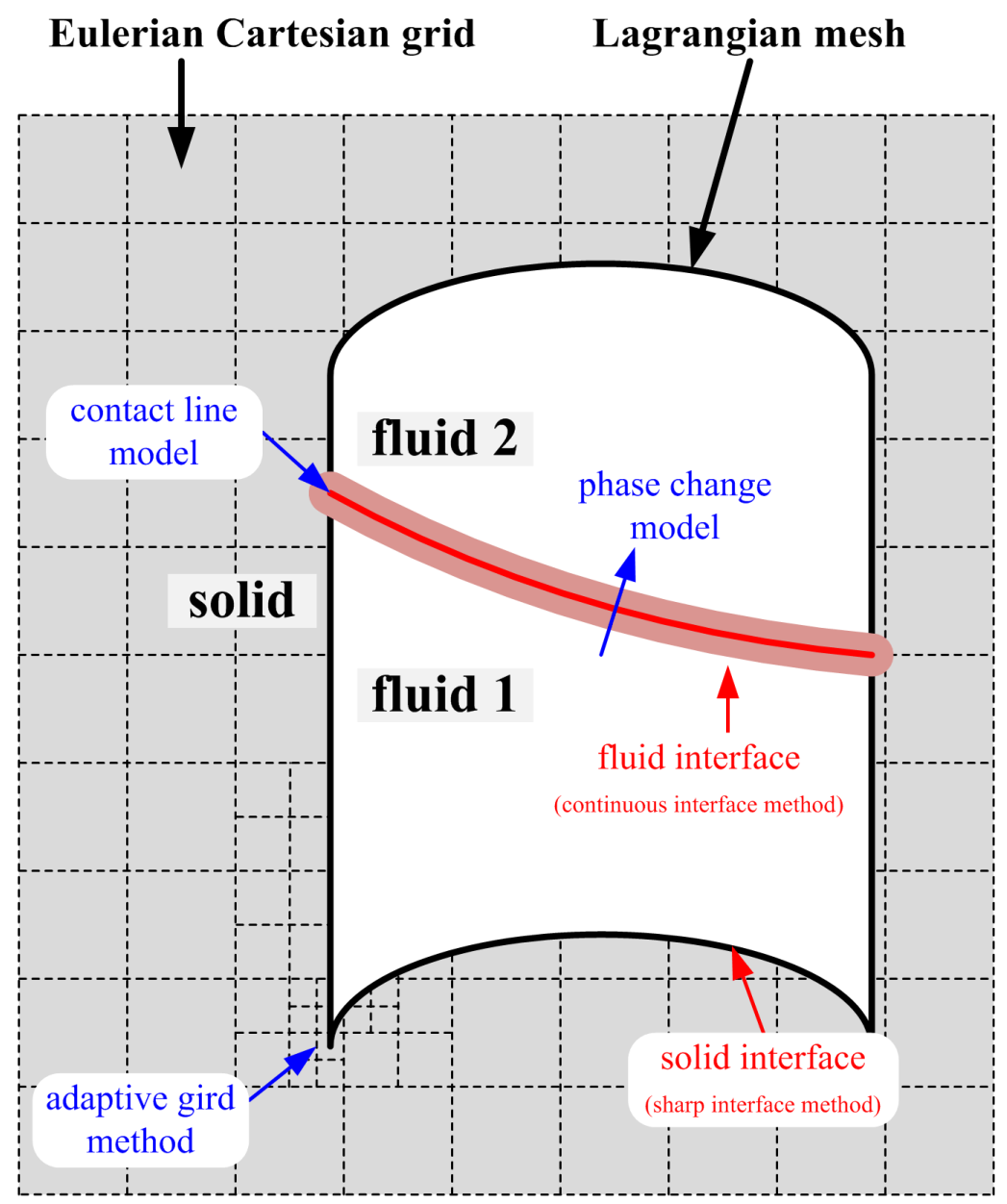

Figure 4. Illustration of the present numerical approach. 


\section{Computational Assessment}

\section{A. Validation of the solid boundary treatment: uniform flow past a circular cylinder}

The simulation of a flow past circular cylinder immersed in a free stream is chosen to verify improvement of the present solid boundary treatment. Computational domain spans 40 times the diameter of cylinder in both $\mathrm{x}$ and $\mathrm{y}$ direction with a cylinder in the center of domain. Slip boundary is applied at the top and bottom of computational domain and non-reflective boundary is specified at the outlet with fixed pressure $\mathrm{P}_{0}$. The spatial boarder provides enough room for pressure field to diffuse without interact with inlet and slip boundary condition too much. Adaptive mesh is used with 2 nd order RKCN convection-diffusion flux scheme. The minimum grid sizes of test cases are $1 / 16 \mathrm{D}, 1 / 32 \mathrm{D}$ and $1 / 64 \mathrm{D}$, where $\mathrm{D}$ denotes the cylinder diameter. Computations perform at Reynolds number 40, 100 and 200. Drag coefficient $C_{D}$, lift coefficient $C_{L}$, pressure coefficient $C p$ and Strouhal number are computed and compared with others.

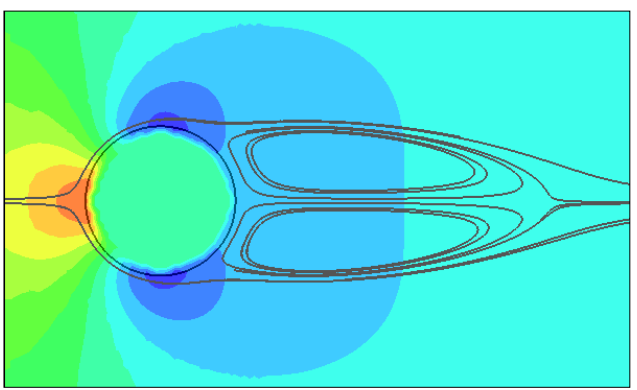

Figure 5. Pressure contour and streamline at $\mathrm{Re}=40$.

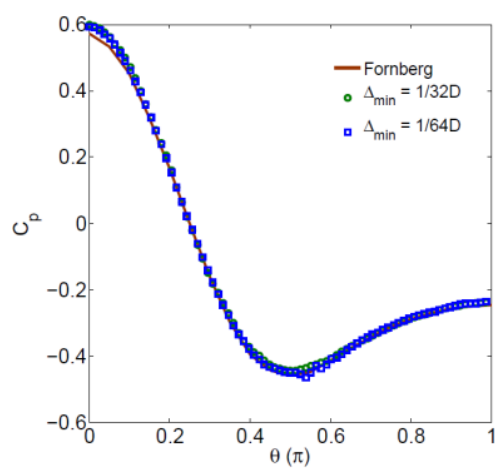

Figure 6. Pressure coefficient at cylinder surface, $\operatorname{Re}=40$.

Figure 5 presents pressure contour and streamlines at Reynolds number 40. Pressure contour lines normal to the solid interface shows that pressure is well approximated by homogeneous Neumann condition. Figure 6 presents the pressure coefficient at the upper half cylinder circumference together with Fornberg's results. ${ }^{29}$ Overall pressure is well resolved with fair grid size. At Reynolds number 100 and 200, the vortex shedding behavior is captured and non-dimensional parameters, Strouhal number and periodic fluctuation of drag and lift coefficient are tracked and plotted in Figure 7. The computed Stroual numbers are 0.164 and 0.192 for Reynolds number 100 and 200 with lift coefficient 0.31 and 0.68 respectively. Our current results agree well with previous studies ${ }^{30,31}$ using immersed boundary method, whose Strouhal number around 0.196 and averaged lift coefficient 0.68 for case of Reynolds number 200. In case of Reynolds number 40, drag coefficient is 1.52 and length of recirculation zone is 2.22 diameter of the circular cylinder, which matches well with Ye at al.'s quadratic interpolation results. ${ }^{17}$

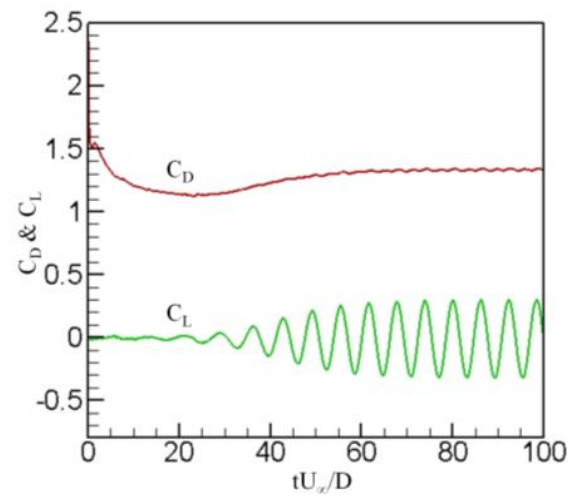

(a)

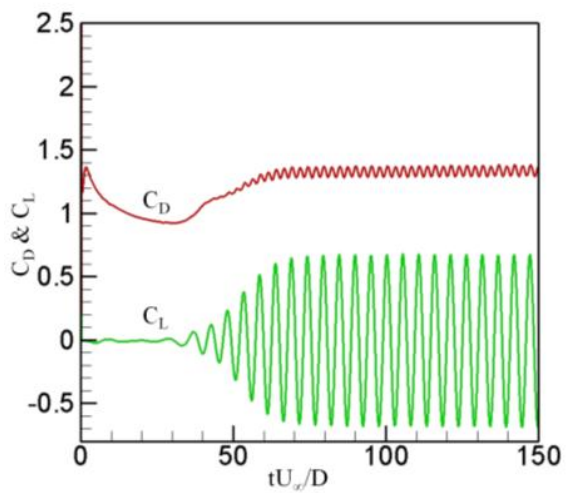

(b)

Figure 7. Lift and drag coefficient. (a) $\operatorname{Re}=100$. (b) $\operatorname{Re}=200$. 


\section{B. Validation of phase change model: 1-D Stefan problem}

A one dimensional phase change test problem is performed to evaluate the present computational approach for phase change. 1-D two-phase Stefan problem with no density effect are first validated and compared with theoretical solution. ${ }^{32}$ The phase change with density effect is further simulated for open and closed container, and selfpressurization in a closed container is measured in a closed container case.

Figure 8 shows the numerical configuration for 1-D two-phase Stefan problem with the same density on a semiinfinite slab, where the left wall was maintained at a constant temperature $T_{0}$ with the interface location, $X(t)$, denoted by separating the vapor and liquid phases on the left and right sides respectively. The initial temperature of phase 2 is a certain value, $T_{\infty}$, but varies in time due to heat transfer from phase 1 . The initial conditions and boundary conditions are summarized in Table 1.

Table 1. One dimensional two-phase Stefan problem

\begin{tabular}{|c|l|}
\hline Interface temperature & $\mathrm{T}(\mathrm{X}(\mathrm{t}), \mathrm{t})=\mathrm{T}_{\text {sat }}, \quad(\mathrm{t}>0)$ \\
\hline Stefan condition & $\rho \mathrm{LX}^{\prime}(\mathrm{t})=-\mathrm{k}_{1} \mathrm{~T}_{\mathrm{x}}\left(\mathrm{X}(\mathrm{t})^{-}, \mathrm{t}\right)+\mathrm{k}_{2} \mathrm{~T}_{\mathrm{x}}\left(\mathrm{X}(\mathrm{t})^{+}, \mathrm{t}\right)$ \\
\hline \multirow{2}{*}{ Initial condition } & $\mathrm{T}(\mathrm{x}, 0)=\mathrm{T}_{\infty}<\mathrm{T}_{\text {sat }}, \quad(\mathrm{x}>0)$ \\
\cline { 2 - 2 } & $\mathrm{X}(0)=0$ \\
\hline \multirow{2}{*}{ Boundary condition } & $\mathrm{T}(0, \mathrm{t})=\mathrm{T}_{0}>\mathrm{T}_{\text {sat }}, \quad(\mathrm{t}>0)$ \\
\cline { 2 - 2 } & $\lim _{\mathrm{x} \rightarrow \infty} \mathrm{T}(\mathrm{x}, \mathrm{t})=\mathrm{T}_{\infty}, \quad(\mathrm{t}>0)$ \\
\hline
\end{tabular}

The theoretical interface location and temperature distribution for two-phase Stefan problem are given by Eqs. (26)-(29), where $\alpha$ and $C$ are thermal diffusivity and heat capacity and $L$ is the latent heat of phase change such as evaporation or melting. Parameter $\lambda$ is obtained by solving the transcendental Eq. (29).

The computations are started with the initial interface location at $\mathrm{X}(\mathrm{t})=0.1$, assuming $T_{0}=1$ at the left end and $T_{\infty}=-1$ at the right end with the saturation temperature $T_{\text {sat }}=0$. The thermal conductivity ratio is varying from 0.1 to 10 . The parameter $\lambda$ is computed based on the solution of transcendental equation for the chosen fluid properties. The theoretical temperature distribution at the time that corresponds to the initial interface location is imposed as the initial condition of the present numerical study, with the computational domain from $\mathrm{x}=0$ to $\mathrm{x}=1$ using 200 grid cells.

$$
\begin{gathered}
X(t)=2 \lambda \sqrt{\alpha t} \\
\left.T(x, t)=T_{0}-\left(\frac{T_{0}-T_{\text {sat }}}{\operatorname{erf}(\lambda)}\right) \operatorname{erf}\left(\frac{x}{2 \sqrt{\alpha_{1} t}}\right), \quad \text { (for phase } 1\right) \\
T(x, t)=T_{\infty}+\left(\frac{T_{\text {sat }}-T_{\infty}}{\operatorname{erfc}\left(\lambda \sqrt{\alpha_{1} / \alpha_{2}}\right)}\right) \operatorname{erfc}\left(\frac{x}{2 \sqrt{\alpha_{2} t}}\right), \quad \text { (for phase 2) } \\
\lambda=\frac{C_{1}\left(T_{0}-T_{\text {sat }}\right)}{L \sqrt{\pi} \exp \left(\lambda^{2}\right) \operatorname{erf}(\lambda)}-\frac{C_{2}\left(T_{\text {sat }}-T_{\infty}\right)}{L \sqrt{\pi} \exp \left(\left(\lambda \sqrt{\alpha_{1} / \alpha_{2}}\right)^{2}\right) \operatorname{erfc}\left(\lambda \sqrt{\alpha_{1} / \alpha_{2}}\right)}
\end{gathered}
$$

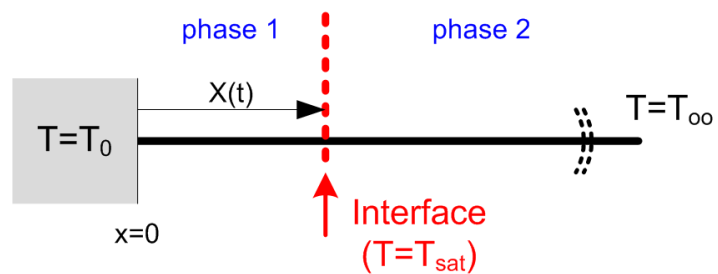

Figure 8. The numerical configuration of one dimensional two-phase change test problem. 


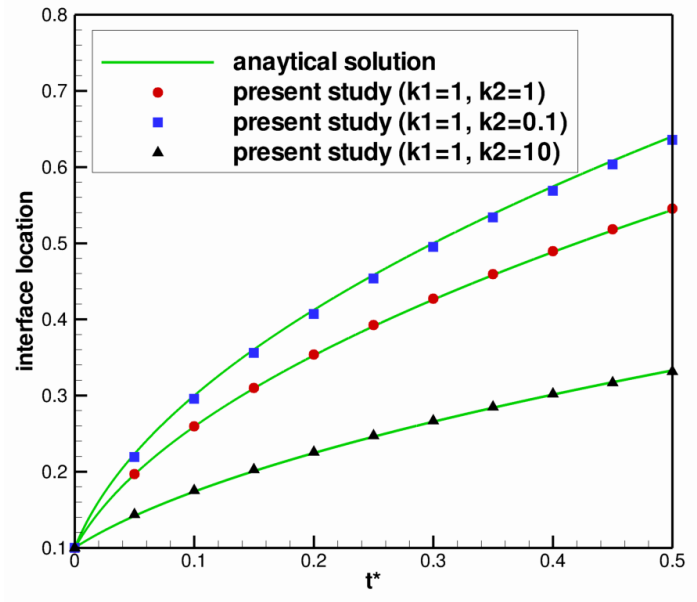

(a)

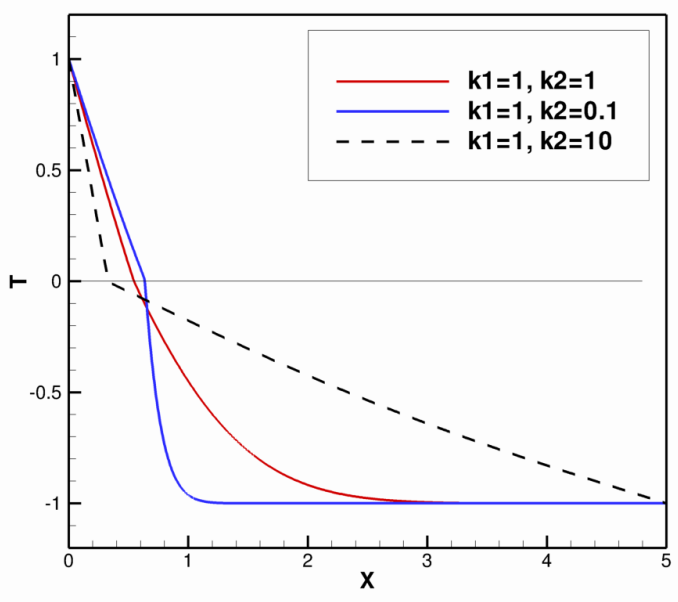

(b)

Figure 9. The comparison of the present numerical study and analytical solution for two-phase Stefan problem. (a) Interface location. (b) Temperature profile at time $=\mathbf{0 . 5}$.

The interface location history during phase change process is shown in Figure 9(a) for the different thermal conductivity ratio from 0.1 to 10 . The interface moves fast in the beginning and slows down as time goes on because the temperature gradient becomes smaller as the interface goes far from the hot temperature at $x=0$. It is known that the continuous interface treatment has some errors in simulating thermal effect. However, Ferziger demonstrated that the error is due to an incorrect smoothed averaging of material properties in the stationary heat conduction problem with no phase change. ${ }^{25}$ In the present study, the thermal conductivity is averaged using in the Eqs. (4)-(7), and it demonstrates substantial agreement with analytical solution, even for phase change cases. The different temperature profile for each case is shown in Figure 9(b), where small thermal conductivity in phase 2 produces a large temperature gradient, and it results in fast movement of interface due to a large mass transfer.

The density effect in a phase change is studied in the open and closed container system. The liquid density is assumed to be twice that of the gas density, and the given material properties and boundary conditions are shown in Table 2. When the liquid phase is transferred into gas phase, the mass of liquid phase is conserved during the phase change according to the rule of mass conservation. However, the volume of transferred phase is not conserved. Because the density ratio of liquid to gas is twice from the beginning, the volume of the transferred gas is initially twice that of the volume of the corresponding liquid. The difference of volume results in a rise of density and pressure in the gas phase, and/or a volume expansion of gas phase. In Figure 10(a), a container open at right end is considered, where the gas phase will expand and the liquid phase will move to right during phase change process. If we assume there is no mechanical drag, the bulk of the liquid will move with no mechanical force, and the density of the gas will be same to the original density as the gas phase expands to the right end. Figure 11(a) shows the interface location change in the phase change process, and the open container simulation is identical with the analytical solution.

The closed container case is shown in Figure 10(b), where the both end are closed, and the liquid cannot move to the right; therefore, the gas cannot be expanded. This causes the pressure to rise in the gas phase of confined container as the self-pressurization occurs in a closed spacecraft fuel tank system. Figure 11(a) shows the interface moves slowly in a closed system since the liquid phase cannot move and the interface movement is completely dominated by the amount of phase change. Figure 11(b) compares the pressure between two systems. In the open container, the liquid moves freely, so the pressure of the gas is maintained. This is the same as it is in the beginning because the expanded gas pushes the liquid up and into the right side. When the phase change occurs in the closed system, the pressure of gas phase rises when density increases. However, the speed of pressure rise decreases in time, and it reaches asymptotic values as the density ratio decreases. 
Table 2. Parameters for 1-D two-phase Stefan problem with density effect.

\begin{tabular}{|c|c|c|}
\hline Parameter & Phase 1 (gas) & Phase 2 (liquid) \\
\hline Temperature at end & $\mathrm{T}_{0}=1.0$ & $\mathrm{~T}_{\infty}=-1.0$ \\
\hline Saturation temperature & \multicolumn{2}{|c|}{$\mathrm{T}_{\text {sat }}=0.0$} \\
\hline density $(\rho)$ & 1.0 & 2.0 \\
\hline heat capacity $(\mathrm{C})$ & 1.0 & 1.0 \\
\hline thermal conductivity $(\mathrm{K})$ & 1.0 & 1.0 \\
\hline
\end{tabular}

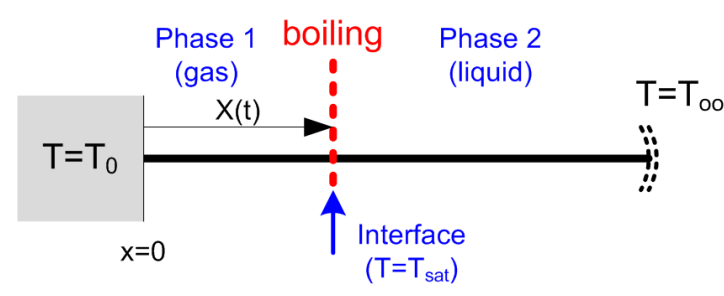

(a)

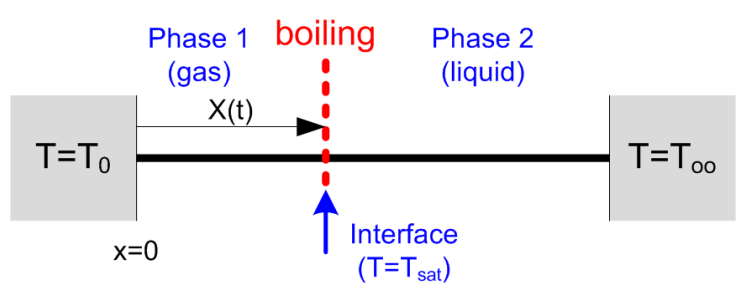

(b)

Figure 10. The numerical configuration of one dimensional Stefan problems with density effect. (a) Open container system with gas expansion and no density change. (b) Closed container with pressure rise.

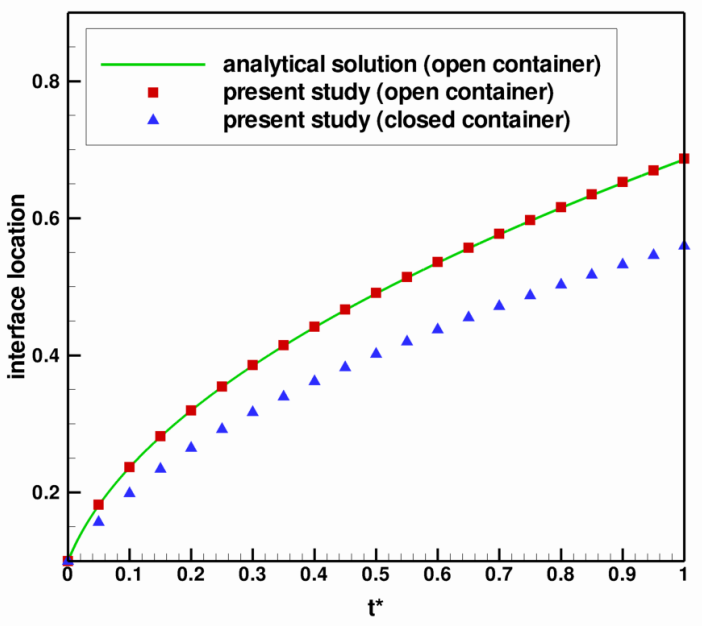

(a)

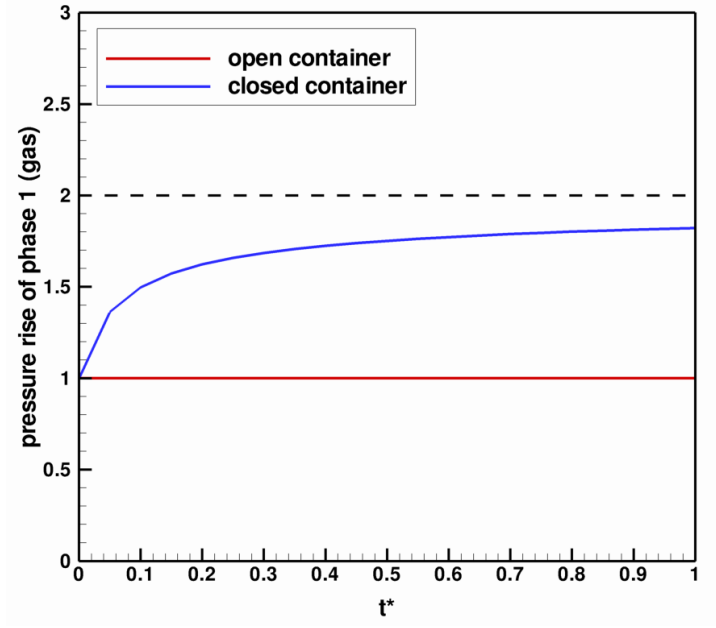

(b)

Figure 11. The comparison of the interface location and the pressure rise between open and closed container system. (a) Interface location. (b) Pressure rise of gas phase. 


\section{2-D melting in a square cavity by natural convection}

Two-dimensional melting driven by natural convection in a square cavity is considered to present the fidelity of the phase-change approach implemented. The simulation is performed in a rectangular tank filled with a pure substance of solid phase, which is suddenly exposed to high isothermal surface at left vertical wall and thermally insulated at top and bottom walls. The initial temperature of the material in this cavity is uniformly $\mathrm{T}_{\mathrm{M}}$ as the temperature of right vertical wall, and gravity is parallel to the vertical wall. Constant material properties are assumed for each phase through the melting process. Figure 12 shows the configuration of a 2-D melting in a square cavity by natural convection flow. The liquid/solid interface is initially located at the left wall, and moves to right by melting process since the heat is transferred by convection and diffusion flow from hot wall of the left side to solid surface. The isothermal temperature is assumed in the solid phase.

In the case of melting driven by natural convection in a square cavity, dimensional analysis shows the heat transfer rate can be expressed in terms power laws of Rayleigh number $\left(R a=g \beta \Delta T L^{3} / \alpha \nu\right)$ at high Prandtl number $(\operatorname{Pr}=v / \alpha)$ range while it is function of $R a$ times $\operatorname{Pr}$ at low Prandtl number range. ${ }^{33,34}$ Here, $g$ is gravitational acceleration, $\beta$ is thermal expansion, $\Delta T$ is the difference of temperature between hot wall and an interface, and $L$ is the height of cavity. $v$ and $\alpha$ are kinematic viscosity and thermal diffusivity, respectively. In this study, two numerical tests of $R a=2.5 \times 10^{4}$ and $2.5 \times 10^{5}$ are simulated at a constant Prandtl number $\operatorname{Pr}=0.02$ and Stefan number $\mathrm{St}=0.01$. Figure 13 shows the location of the interface between liquid and solid phase with temperature contour for $R a=2.5 \times 10^{5}$. The buoyancy force stir the flow field to generate multiple recirculation cells at the beginning of melting and circulations gradually merge into a dominated one when interface come to the middle of cavity. The time history of interface location is similar to the benchmark work reported by Bertrand et. al. ${ }^{34}$ The evolution of the averaged Nusselt number on the hot wall and liquid fraction are presented in Figure 14. In the case of $R a=2.5 \times 10^{4}$, the Nusselt number matches with Lacroix's work. High frequency fluctuation of Nusselt number is observed for case of $R a=2.5 \times 10^{5}$, which is similar to the results captured by Le Quéré. ${ }^{34}$ The oscillatory behavior may be due to the repeat of production and merge of recirculation cells. The liquid fraction shows consistent trend with respect to Lacroix's work at $R a=2.5 \times 10^{4}$, and to Gobin's work at $R a=2.5 \times 10^{5}$.

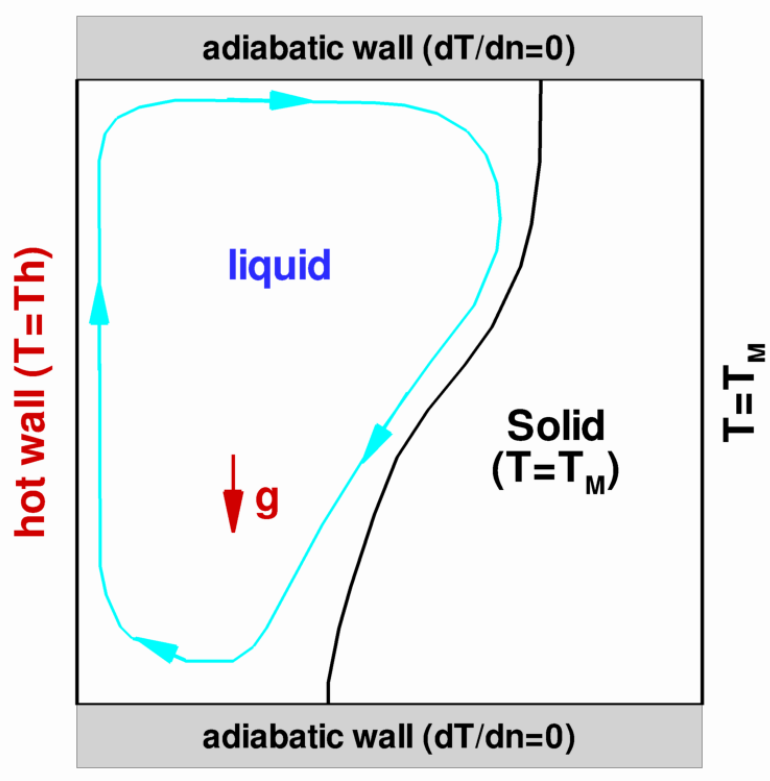

Figure 12. The configuration of a 2-D melting in a square cavity by natural convection flow. 

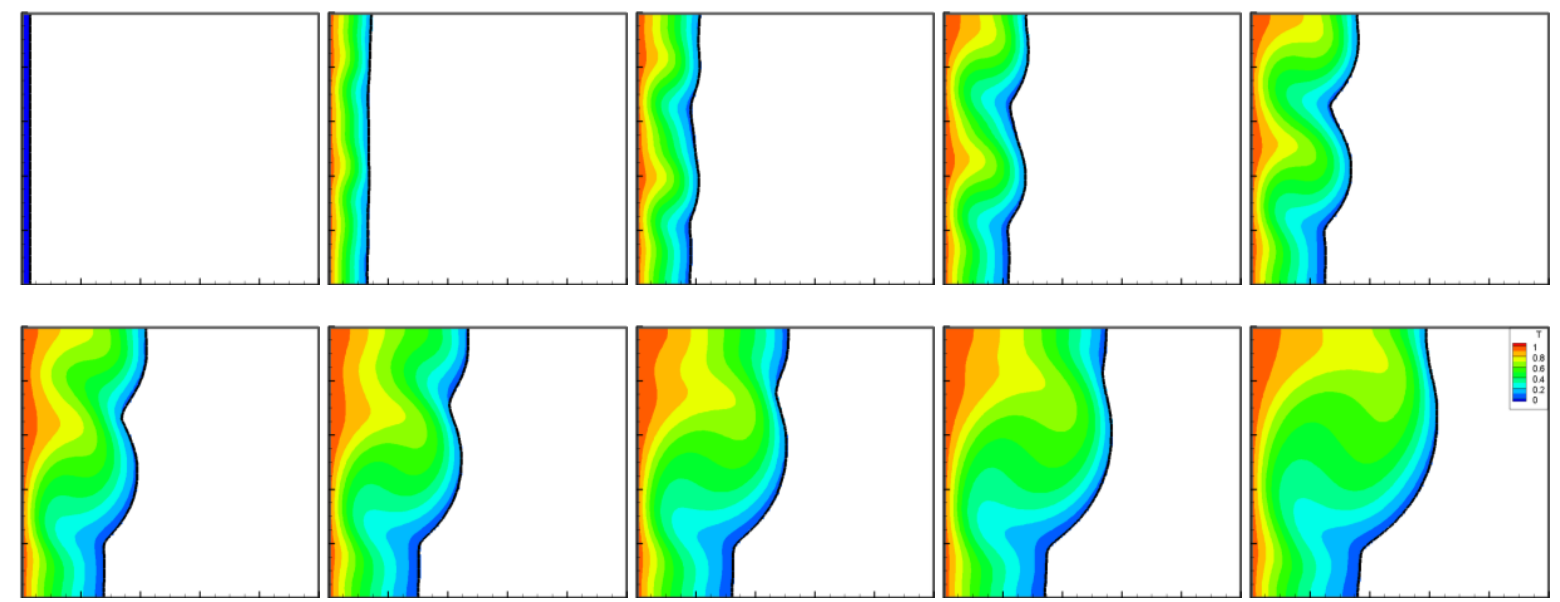

Figure 13. The snapshots of the temperature contours and velocity fields during melting process in a square cavity by natural convection flow at $R a=2.5 \times 10^{5}$.

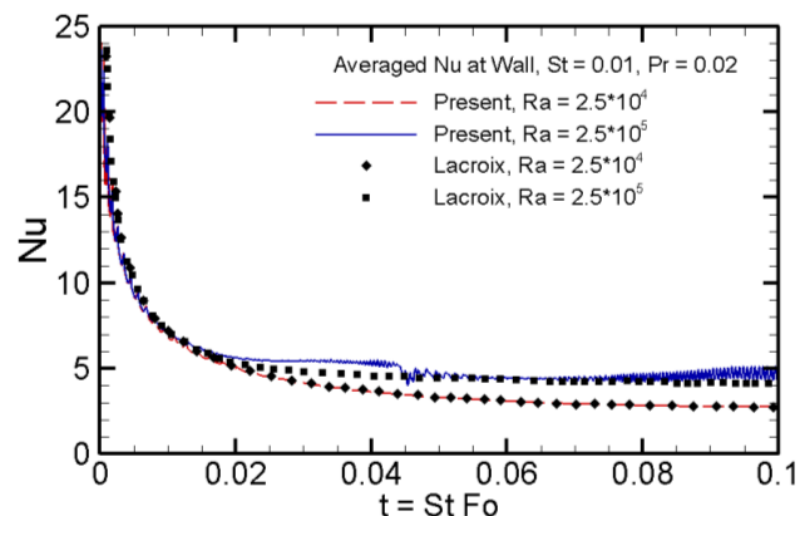

(a)

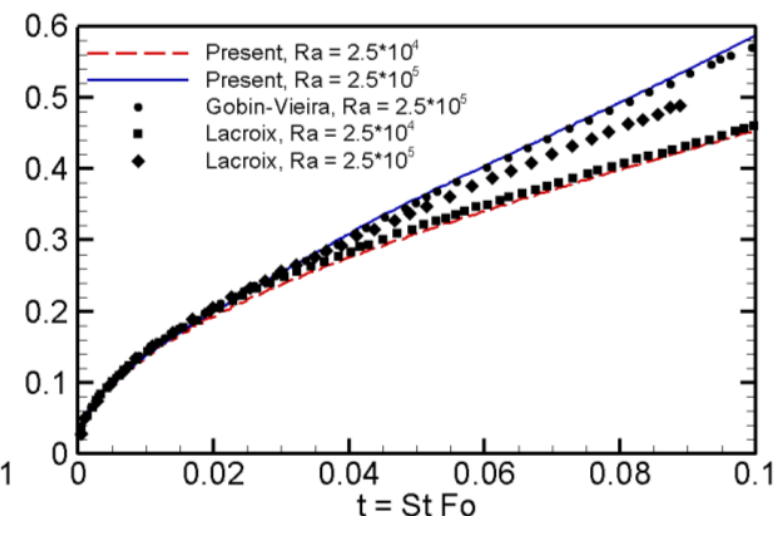

(b)

Figure 14. The comparison of (a) averaged Nusselt number at hot wall and (b) the liquid phase fraction in a melting process by natural convection. 


\section{Self-pressurization in a liquid fuel tank}

The self-pressurization in a cylindrical liquid hydrogen fuel tank is considered. Figure 15(a) shows the geometric configuration of the test case. The ground test at normal gravity is assumed since the interface can be more distorted and the liquid position and vapor bubble distributions are usually unknown under microgravity condition. The axisymmetric numerical computation in Figure 15(b) is conducted due to its effective computation in order to establish a guideline on the amount of self-pressurization at a given heat leak.

First, two different numerical approaches are tested and compared with each other in order to ascertain the role of transport phenomena in the pressurization process of a liquid fuel tank; conduction-only solution with no flow motion and full Navier-Stokes and energy equation solution. For studying the influence of heat distribution, gas-only heating, uniform heating and liquid-only heating cases are simulated with 50\% fill level in Figure 15(b). The study on the influence of liquid fill-level is followed for uniform heating case with three different fill-levels of $25 \%, 50 \%$ and $75 \%$. The total heat power input is fixed at $\mathrm{Q}_{\mathrm{T}}=4.71 \mathrm{~mW}$ with $\mathrm{q}_{\mathrm{H}}=0.1 \mathrm{~W} / \mathrm{m}^{2}$ for uniform heating case and $\mathrm{q}_{\mathrm{H}}=0.2$ $\mathrm{W} / \mathrm{m}^{2}$ for gas-only and liquid-only heating cases. The used material properties at $\mathrm{T}=20.369 \mathrm{~K}$ are summarized in the Table 3.

Table 3. Material properties at $\mathrm{T}=20.369 \mathrm{~K}$.

\begin{tabular}{|l|c|c|}
\hline & Vapor hydrogen & Liquid hydrogen \\
\hline Density, $\rho\left[\mathrm{kg} / \mathrm{m}^{3}\right]$ & 1.3322 & 70.850 \\
\hline Thermal conductivity, $\mathrm{k}[\mathrm{J} / \mathrm{m} \cdot \mathrm{s} \cdot \mathrm{K}]$ & 0.017085 & 0.10382 \\
\hline Dynamic viscosity, $\mu[\mathrm{kg} / \mathrm{m} \cdot \mathrm{s}]$ & $1.0786 \times 10^{-6}$ & $1.3320 \times 10^{-5}$ \\
\hline Specific heat, $\mathrm{C}_{\mathrm{p}}[\mathrm{J} / \mathrm{kg} \cdot \mathrm{K}]$ & $1.2036 \times 10^{4}$ & $1.772 \times 10^{3}$ \\
\hline Thermal diffusivity, $\alpha\left[\mathrm{m}^{2} / \mathrm{s}\right]$ & $2.56 \times 10^{-6}$ & 0.0168 \\
\hline Thermal expansion coefficient, $\beta\left[\mathrm{K}^{-1}\right]$ & 0.04909 & \multicolumn{2}{|c|}{$1.9452 \times 10^{-3}$} \\
\hline Surface tension, $\sigma[\mathrm{N} / \mathrm{m}]$ & \multicolumn{2}{|c|}{$20.356 \times 10^{5}$} \\
\hline Latent heat, $L[\mathrm{~J} / \mathrm{kg}]$ & \multicolumn{2}{|c|}{$1.01400 \times 10^{5}$} \\
\hline Normal boiling temperature, $T_{B}[\mathrm{~K}]$ & \multicolumn{2}{|c|}{} \\
\hline Normal saturation pressure, $P_{S}[\mathrm{~Pa}]$ & & \\
\hline
\end{tabular}

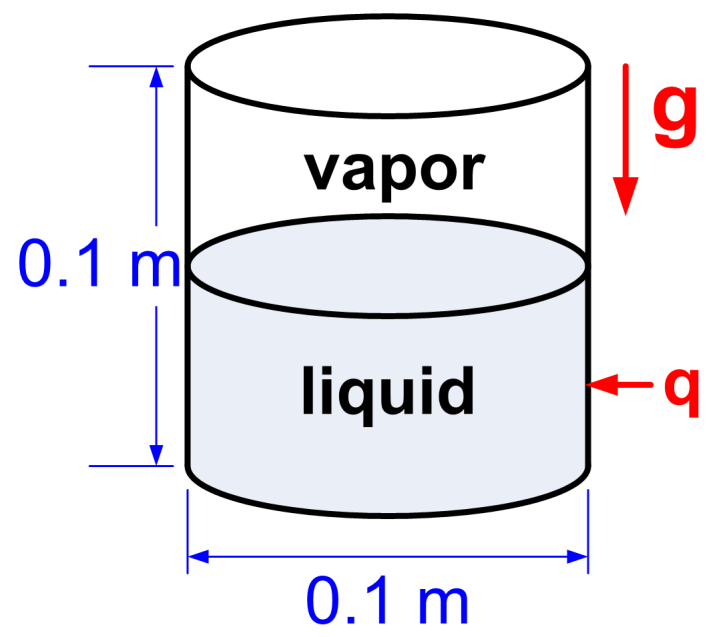

(a)

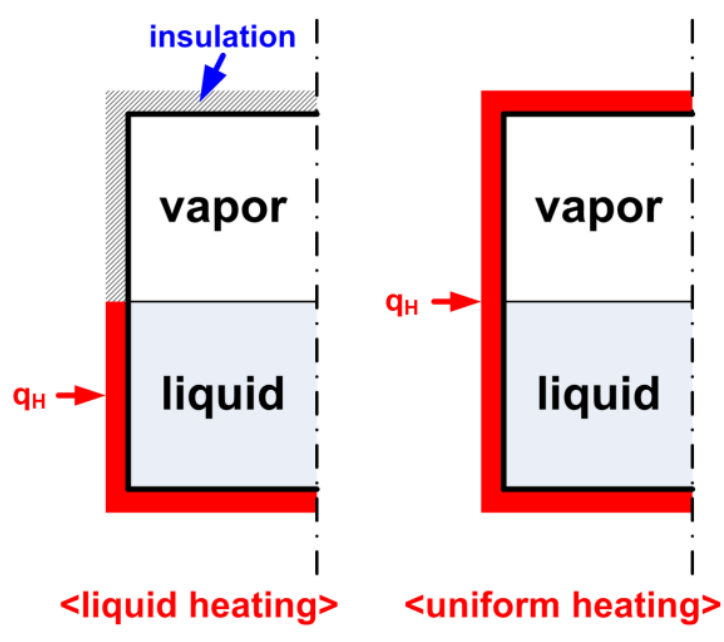

(b)

Figure 15. The numerical configuration. (a) Fuel tank geometry. (b) Axisymmetric computational domain with different tank-wall heat distributions. 
Figure 16 shows the comparison of temperature contour and streamlines between conduction-only solution with no flow motion and full Navier-Stokes/energy equation solution. The uniform heating and $50 \%$ of liquid fill-level is assumed. During a phase change process like a boiling, the amount of the mass transfer and following vapor pressurization is determined by the temperature distribution around the interface. In the conduction-only solution of Figure 16(a), the heat stays on the heated wall, and the temperature around interface is maintained low due to less heat transfer from the wall. However, in the full Navier-Stokes/energy equation solution, the convection flow as well as the conduction transfer heat from the wall into the interface due to natural convection by buoyancy force, which develops especially thin warm liquid layer along the wall in Figure 16(b). This makes in a huge difference in the pressure rise in Figure 17(a), where, less heat transfer of conduction-only solution due to the absence of convection transport phenomena results in underestimated pressure rise. Figure 17(b) shows clearly the difference of the temperature distribution along the centerline axis. This comparison shows that the full Navier-Stokes and energy equation solution is required for the accurate numerical study on the pressure rise in a liquid fuel tank.

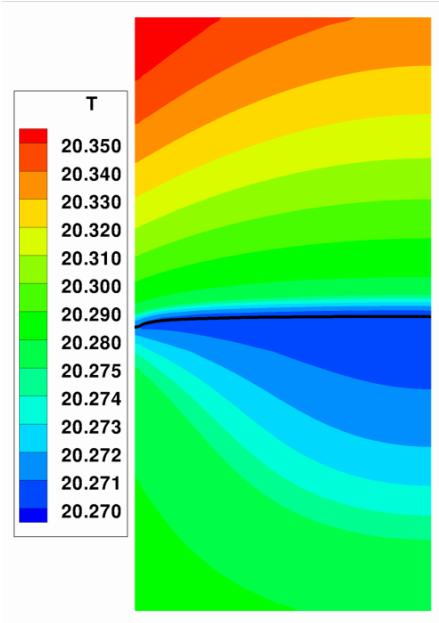

(a)

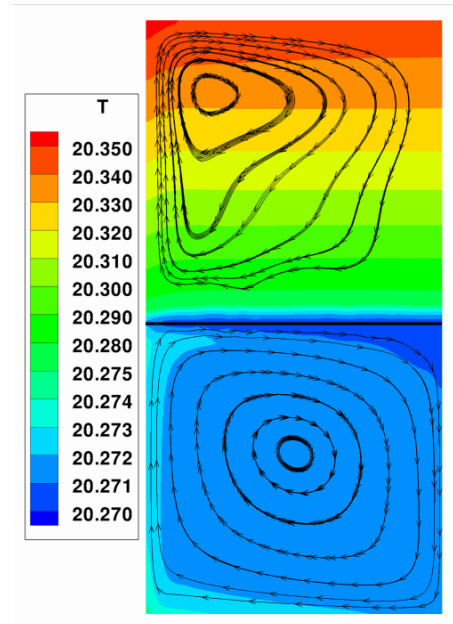

(b)

Figure 16. Temperature contour and streamlines in a fuel tank. (a) conduction-only solution (b) full Navier-Stokes and energy solution.

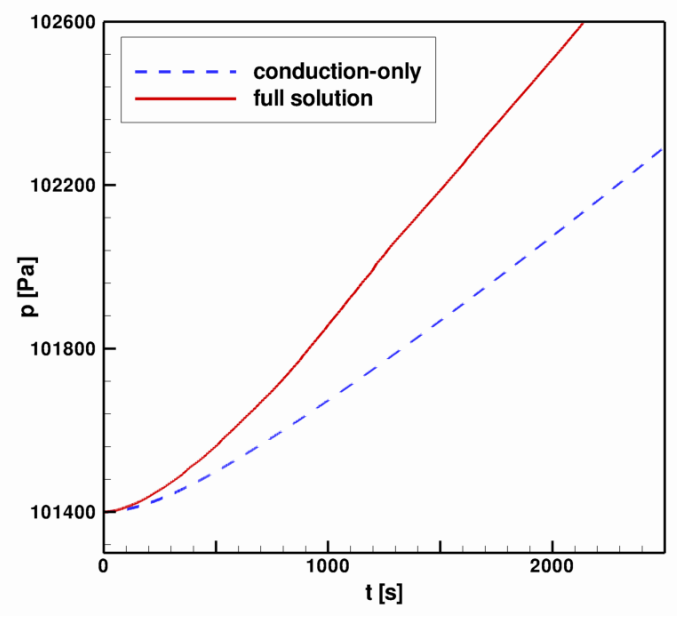

(a)

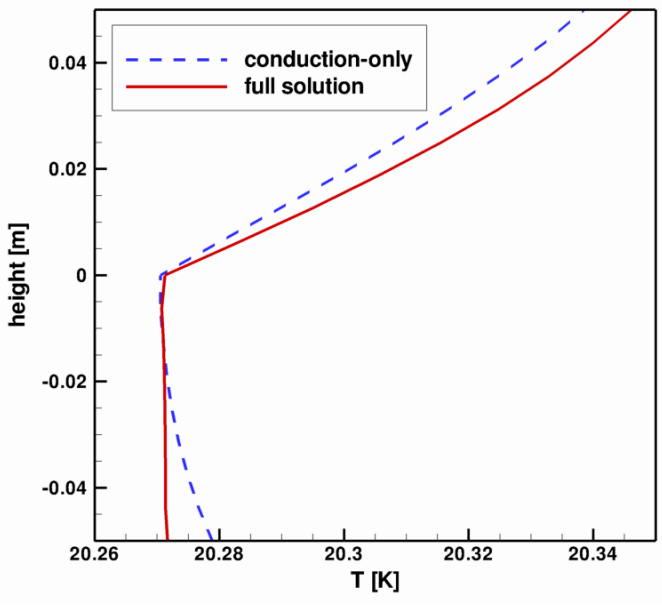

(b)

Figure 17. The comparison of the fuel tank pressurization between conduction-only solution and full Navier-Stokes/energy equation solution. (a) The saturation pressure change in a fuel tank. (b) The temperature profiles along the centerline axis of a fuel tank. 
The effects of heat distribution are studied with three different heat conditions; gas-only heating, uniform heating and liquid-only heating. The same total heat power of $4.71 \mathrm{~mW}$ and $50 \%$ liquid fill-level are assumed for all three cases. In the saturation pressure change of Figure 18(a), the gas-only heating case shows the highest pressure rise, and liquid-only heating results in less pressure rise. The pressure rise rate of Figure 18(b) shows it more clearly. The higher pressure rise rate is observed in the gas-only heating, especially in the beginning. However, the pressure rise rate becomes similar as time goes. It shows that the transport phenomena in the vapor region are important due to shorter time scale of vapor region to understand the pressurization, especially in the beginning of pressurization. The study on the effects of liquid fill-level is followed with three different levels. The higher fill level shows higher pressure rise in the beginning, but it does generally lower pressure rise in Figure 19(a). The pressure rise rate of Figure 19(b) shows that 75\% fill-level has dramatic rise in the beginning due to the fast heat transport in a smaller gas-region. However, the influence decreases quickly, and all three cases show similar pressure rise rates. The longer simulation is needed for considering the influence from liquid region as well as vapor region.

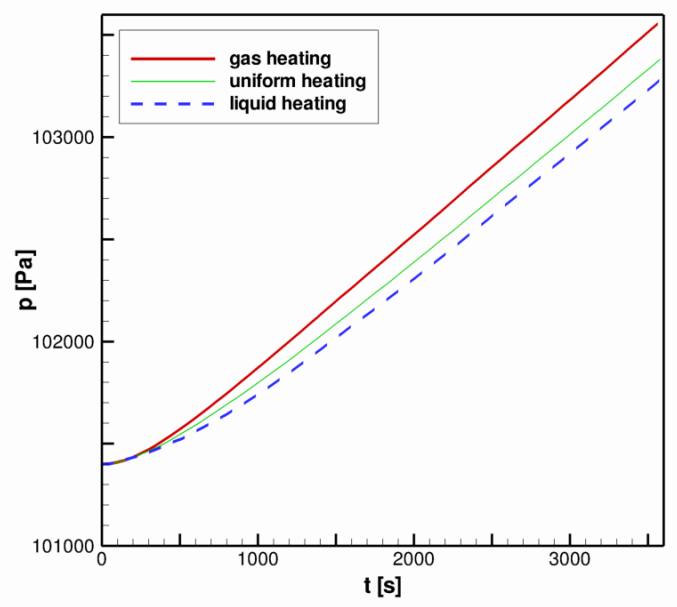

(a)

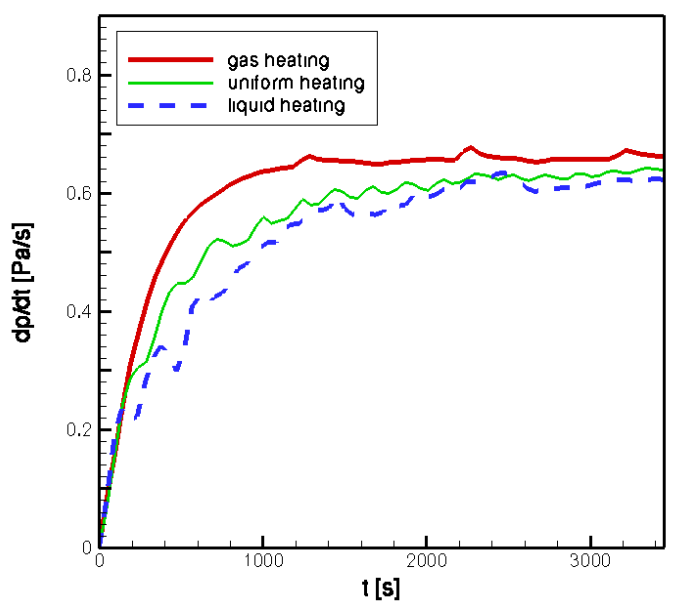

(b)

Figure 18. The effects of heat distributions for fuel tank pressurization. (a) The saturation pressure change in a fuel tank. (b) The saturation pressure rise rates.

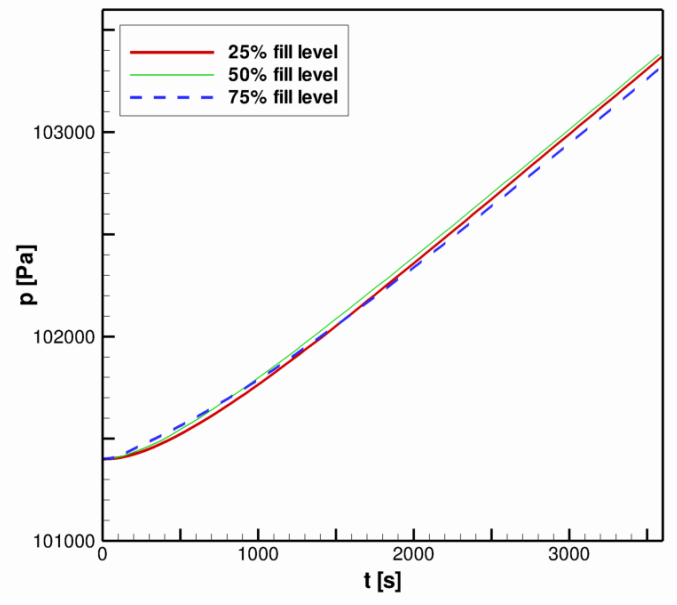

(a)

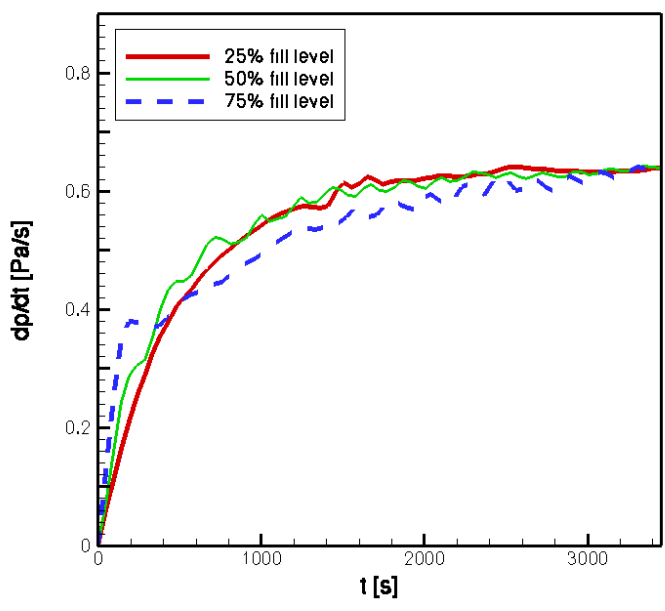

(b)

Figure 19. The effects of liquid hydrogen fill-level for fuel tank pressurization. (a) The saturation pressure in a fuel tank. (b) The saturation pressure rise rates. 


\section{Summary and Conclusions}

In this paper, a 3-D adaptive Eulerian-Lagrangian method for multiphase flow computation is further developed with phase change model for the simulation of spacecraft fuel tank self-pressurization. The governing equations are solved on an Eulerian Cartesian grid with a dynamic local grid adaptation for appropriate resolution. The Lagrangian triangulated surface meshes are implemented for tracking interfaces including different fluid phase boundary and arbitrary shaped solid geometries. The phase change computation is computed from the Stefan condition using temperature gradient across an interface, and is implemented easily with both present continuous fluid/fluid interface framework and sharp solid/fluid interface framework. Numerous test cases have been adopted to evaluate the performance of the modeling and computational capabilities of the present approach, including:

(1) Uniform flow past a circular cylinder,

(2) 1-D two-phase Stefan problem including density effects,

(3) 2-D melting in a square cavity by natural convection,

(4) Self-pressurization in a cylindrical liquid fuel tank.

Specifically, the following observations can be made in the fuel tank self-pressurization case:

(1) The transport phenomena play an important role in the self-pressurization of a liquid fuel tank, and conduction-only solution underestimates the pressure rise,

(2) The heat transfer in the vapor region has a large influence on the pressurization, especially in the beginning,

(3) The full Navier-Stokes and energy equation solution is required to respect the heat transfer via both convection and conduction in both vapor and liquid phases.

We are in the process of further refining and assessing the computational techniques along with detailed code verification and validation exercises for practical engineering problems involving spacecraft thermal stratification and self-pressurization from normal to micro-gravity condition with various heat distributions and jet mixing conditions.

\section{Acknowledgments}

The work reported in this paper has been partially supported by NASA Constellation University Institutes Program (CUIP), Claudia Meyer and Jeff Rybak program managers. We have benefited from the discussion with John Peugeot and Jeff West of NASA Marshall Space Flight Center.

\section{References}

${ }^{1}$ Hastings, L.J., Plachta, D.W., Salerno, L., and Kittel, P., "An overview of NASA efforts on zero boiloff storage of cryogenic propellants," Cryogenics, Vol. 41, No. 11-12, 2001, pp. 833 - 839.

${ }^{2}$ Aydelott, J.C., "Effect of gravity on self-pressurization of spherical liquid-hydrogen tankage," NASA-TN-D-4286, 1967.

${ }^{3}$ Poth, L.J. and van Hook, J.R., "Control of the Thermodynamic State of Space-Stored Cryogens by Jet Mixing," Journal of Spacecraft and Rockets, Vol. 9, No. 5, 1972, pp. 332-336.

${ }^{4}$ Aydelott, J.C. and Spuckler, C.M., "Effect of size on normal-gravity self-pressurization of spherical liquid hydrogen tankage," NASA-TN-D-5196, 1969.

${ }^{5}$ Aydelott, J.C., "Modeling of space vehicle propellant mixing," NASA-TP-2107, 1983.

${ }^{6}$ Lin, C.S., Hasan, M.M., and Van Dresar, N.T., "Experimental investigation of jet-induced mixing of a large liquid hydrogen storage tank." 6th AIAA/ASME, Joint Thermophysics and Heat Transfer Conference, Colorado Springs, CO, 1994, AIAA-19942079.

${ }^{7}$ Vaughan, D.A. and Schmidt, G.R., "Analytical modeling of no-vent fill process," Journal of Spacecraft and Rockets, Vol. 28, No. 5, 1991, pp. 574-579.

${ }^{8}$ Cha, Y.S., Niemann, R.C., and Hull, J.R., "Thermodynamic analysis of helium boil-off experiments with pressure variations," Cryogenics, Vol. 33, No. 7, 1993, pp. 675-679.

${ }^{9}$ Navickas, J., "Prediction of a liquid tank thermal stratification by a finite difference computing method." 24th ASME, SAE, and ASEE, Joint Propulsion Conference, Boston, MA, 1988, AIAA-1988-2917.

${ }^{10}$ Lin, C.S. and Hasan, M.M., "Numerical investigation of the thermal stratification in cryogenic tanks subjected to wall heat flux." 28th AIAA Aerospace Science Meeting, Reno, NV, 1990, AIAA-1990-0354.

${ }^{11}$ Grayson, G.D. and Navickas, J., "Interaction between fluid-dynamic and thermodynamic phenomena in a cryogenic upper stage." AlAA 28th Thermophysics Conference, Orlando, FL, 1993, AIAA-1993-2753.

${ }^{12}$ Shyy, W., Udaykumar, H.S., Rao, M.M., and Smith, R.W., Computational Fluid Dynamics with Moving Boundaries, Taylor \& Francis, Philadelphia, 1996. 
${ }^{13}$ Prosperetti, A. and Tryggvason, G., Computational Methods for Multiphase Flow, Cambridge University Press, New York, 2007.

${ }^{14}$ Peskin, C.S., "The immersed boundary method," Acta Numerica, Vol. 11, 2003, pp. 479-517.

${ }^{15}$ Tryggvason, G., Bunner, B., Esmaeeli, A., Al-Rawahi, N., Tauber, W., Han, J., Jan, Y.J., Juric, D., and Nas, S., "A fronttracking method for the computations of multiphase flow," Journal of Computational Physics, Vol. 169, No. 2, 2001, pp. 708759.

${ }^{16}$ Singh, R. and Shyy, W., "Three-dimensional adaptive Cartesian grid method with conservative interface restructuring and reconstruction," Journal of Computational Physics, Vol. 224, No. 1, 2007, pp. 150-167.

${ }^{17}$ Ye, T., Mittal, R., Udaykumar, H.S., and Shyy, W., "An accurate Cartesian grid method for viscous incompressible flows with complex immersed boundaries," Journal of Computational Physics, Vol. 156, No. 2, 1999, pp. 209-240.

${ }^{18}$ Ye, T., Shyy, W., and Chung, J.N., "A fixed-grid, sharp-interface method for bubble dynamics and phase change," $J$. Comput. Phys., Vol. 174, No. 2, 2001, pp. 781-815.

${ }^{19}$ Mittal, R., Dong, H., Bozkurttas, M., Najjar, F.M., Vargas, A., and von Loebbecke, A., "A versatile sharp interface immersed boundary method for incompressible flows with complex boundaries," Journal of Computational Physics, Vol. 227, No. 10, 2008, pp. 4825-4852.

${ }^{20}$ Son, G. and Dhir, V.K., "Numerical Simulation of Film Boiling Near Critical Pressures With a Level Set Method," Journal of Heat Transfer, Vol. 120, No. 1, 1998, pp. 183-192.

${ }^{21}$ Welch, S.W.J. and Wilson, J., "A Volume of Fluid Based Method for Fluid Flows with Phase Change," Journal of Computational Physics, Vol. 160, No. 2, 2000, pp. 662-682.

${ }^{22}$ Juric, D. and Tryggvason, G., "Computations of boiling flows," International Journal of Multiphase Flow, Vol. 24, No. 3, 1998, pp. 387-410.

${ }^{23}$ Shin, S. and Juric, D., "Modeling three-dimensional multiphase flow using a level contour reconstruction method for front tracking without connectivity," Journal of Computational Physics, Vol. 180, No. 2, 2002, pp. 427-470.

${ }^{24}$ Luo, X.-Y., Ni, M.-J., Ying, A., and Abdou, M.A., "Numerical Modeling for Multiphase Incompressible Flow with Phase Change," Numerical Heat Transfer, Part B: Fundamentals, Vol. 48, No. 5, 2005, pp. 425-444.

${ }^{25}$ Ferziger, J.H., "Interfacial transfer in Tryggvason's method," International Journal for Numerical Methods in Fluids, Vol. 41, No. 5, 2003, pp. 551-560.

${ }^{26}$ Uzgoren, E., Sim, J., and Shyy, W., "Marker-based, 3-D adaptive Cartesian grid method for multiphase flow around irregular geometries," Communications in Computational Physics, Vol. 5, No. 1, 2009, pp. 1-41.

${ }^{27}$ Sim, J. and Shyy, W., "Interfacial flow computations using adaptive Eulerian-Lagrangian method for spacecraft applications," International Journal for Numerical Methods in Fluids, 2010.

${ }^{28}$ Kim, J., Kim, D., and Choi, H., "An immersed-boundary finite-volume method for simulations of flow in complex geometries," Journal of Computational Physics, Vol. 171, No. 1, 2001, pp. 132-150.

${ }^{29}$ Fornberg, B., "A numerical study of steady viscous flow past a circular cylinder," Journal of Fluid Mechanics, Vol. 98, 1980, pp. 819-855.

${ }^{30}$ Linnick, M.N. and Fasel, H.F., "A high-order immersed interface method for simulating unsteady incompressible flows on irregular domains," Journal of Computational Physics, Vol. 204, No. 1, 2005, pp. 157-192.

${ }^{31}$ Taira, K. and Colonius, T., "The immersed boundary method: A projection approach," Journal of Computational Physics, Vol. 225, No. 2, 2007, pp. 2118-2137.

${ }^{32}$ Alexiades, V. and Solomon, A.D., Mathematical Modeling of Melting and Freezing Processes, Taylor \& Francis, 1993.

${ }^{33}$ Jany, P. and Bejan, A., "Scaling theory of melting with natural convection in an enclosure," International Journal of Heat and Mass Transfer, Vol. 31, No. 6, 1988, pp. 1221-1235.

${ }^{34}$ Bertrand, O., Binet, B., Combeau, H., Couturier, S., Delannoy, Y., Gobin, D., Lacroix, M., Quere, P.L., Medale, M., Mencinger, J., Sadat, H., and Vieira, G., "Melting driven by natural convection A comparison exercise: first results," International Journal of Thermal Sciences, Vol. 38, 1999, pp. 5-26. 